\title{
Ward pharmacy: a foundation for prescribing audit?
}

\author{
R Batty, N Barber
}

\begin{abstract}
Objectives - To determine the extent and nature of prescription monitoring incidents by hospital pharmacists and to derive a performance indicator to allow prescription monitoring to be compared among hospitals in North West Thames region.
\end{abstract}

Design - Survey of all self recorded prescription monitoring incidents for one week in June 1990.

Setting - All (31) acute hospitals in the region with pharmacy departments on site, covering 10337 beds.

Subjects - 210 pharmacists.

Main measures - Number of prescription monitoring incidents recorded, their nature, and outcome; a performance indicator of prescription monitoring (incidents/100 beds/week) and its variation according to specialty and site.

Results - 3273 prescription monitoring incidents were recorded (median 89 per hospital, range 3-301), the most common being related to the dose and frequency of administration of the drug (933 incidents, $29 \%$ ). These incidents led to alterations of prescriptions on 1611 occasions; the pharmacist's advice was rejected on 81 . The greatest number of prescription monitoring incidents $/ 100$ beds/week by specialty was recorded for intensive therapy units (median 75); the medians for medicine and surgery were 32 and 21 respectively. This performance indicator varied 20 -fold when analysed by site, values ranging from 3.6 to 82.1 (median 29.8).

Conclusions - Hospital pharmacists play a large part in monitoring and improving prescribing, and most of their interventions are related to the basics of prescribing. They therefore have a role in medical audit, working with clinicians to identify prescribing problems, and to set standards and monitor practice. $A$ performance indicator of prescription monitoring incidents/100 beds/week allows comparison of pharmacists' activities among sites and may be a valuable tool in auditing them.

\section{Introduction}

Clinical pharmacy has been described in the United Kingdom as a "developing role in which pharmaceutical skills are systematically applied to medicine usage, both at the policy making level and in the treatment of individual patients." For many years it has been thought of as promoting the safe, effective, and economic use of medicines; more recently it has also been suggested that pharmacists should also consider the patient's quality of life. ${ }^{2}$ In the hospital service in the United Kingdom clinical pharmacy evolved from ward pharmacy visiting services developed in the late $1960 \mathrm{~s}$; routine prescription monitoring is part of clinical pharmacy and is now common in most hospitals. A structured approach to prescription monitoring has been described by several authors. ${ }^{3-5}$ There are few published studies describing monitoring activities and the interventions by pharmacists that result from them; all these studies have limitations. Most have been conducted in the United States (as recently reviewed by Klopfer and Einarson ${ }^{6}$ ), but American pharmacists' clinical activities are not directly comparable to those of pharmacists in the United Kingdom. In the United Kingdom studies have been restricted to individual hospitals or districts. ${ }^{7-10}$ As pharmacy is organised at a district level it is difficult to generalise about prescribing or pharmacists' activities from these studies. The collection and review of clinical pharmacists' interventions has been suggested as part of a quality assurance programme; ${ }^{10}{ }^{11}$ the focus, however, has been on management of local pharmacy services.

As a regional unit our study has two objectives. Firstly, to study the nature and extent of pharmacists' interventions as a consequence of monitoring prescriptions; among other things this could be an indicator of prescribers' problems and a stimulus to medical audit. Secondly, to determine whether a performance indicator could be devised which would be a marker of the extent of clinical pharmacy activities in the wards.

\section{Methods}

All 31 acute hospitals in the North West Thames region which had pharmacies on site were included in the survey. For seven consecutive days in June 1990 pharmacy staff in these hospitals recorded all prescription monitoring incidents (PMIs). A PMI was defined as any incident, usually triggered by reading the prescription, which caused the pharmacist to doubt the appropriateness of a prescription and to take further action to ensure that the prescription was appropriate. Pharmacists' annotations to prescription sheets (for example, adding the generic name of a drug or clarifying instructions) were not 


\section{North West Thames region Pharmaceutical Service problem identification record form for inpatient prescriptions}

Please tick column as appropriate, or enter abbreviation described in notes on reverse.

Hospital ................................. Name (note a)

Problems identified in dispensary or in wards (delete as applicable)

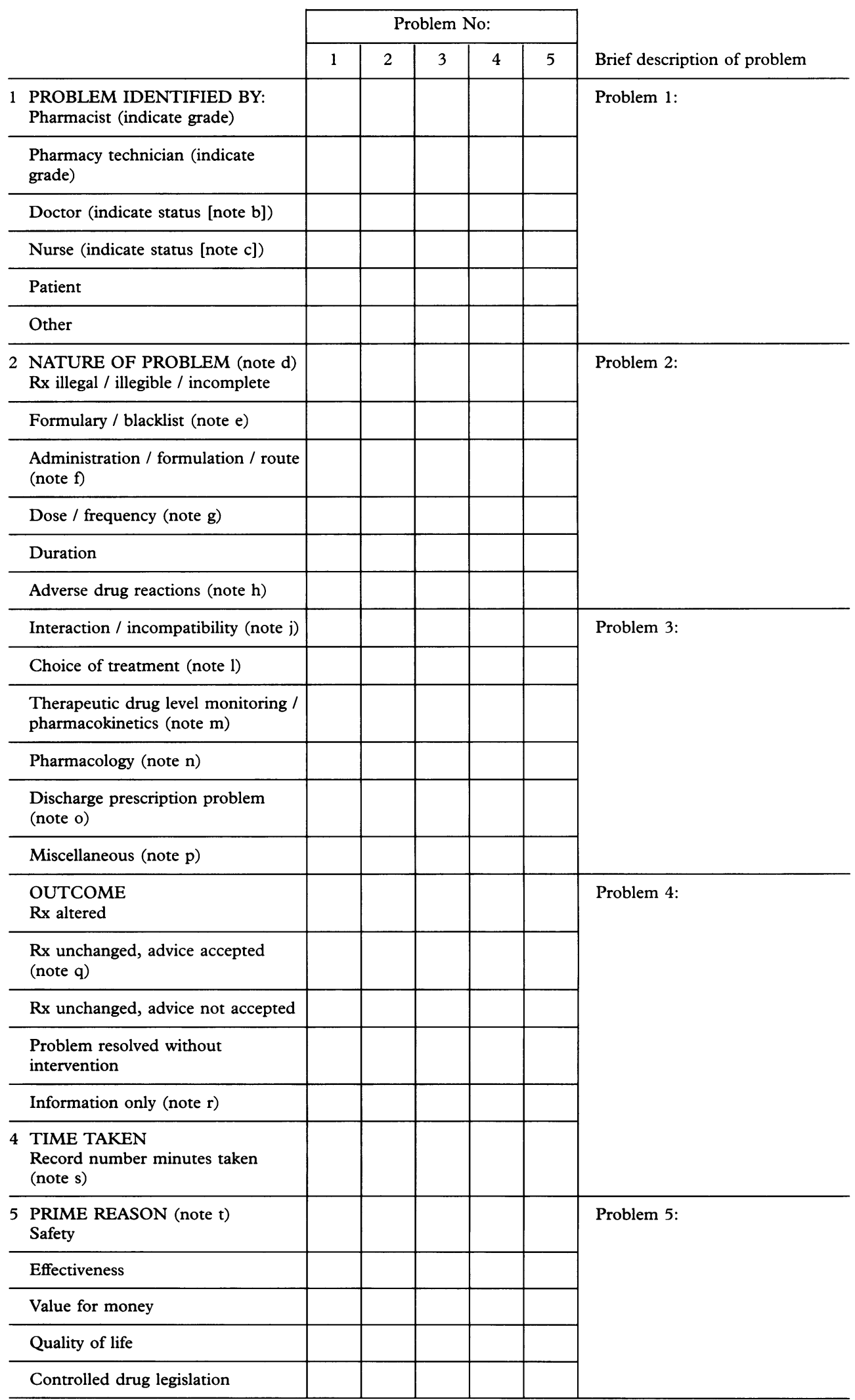


Table 1 Nature of 3273 prescription monitoring incidents identified in 31 hospitals in North West Thames region, June 1990

\begin{tabular}{lcc}
\hline Nature of incident & No & $(\%)^{\star}$ \\
\hline Dose or frequency & 933 & $(29)$ \\
Choice of treatment & 468 & $(14)$ \\
Administration or formulation or & 447 & $(14)$ \\
$\quad$ route & 339 & $(10)$ \\
Discharge prescription problem & 294 & $(9)$ \\
Prescription illegal or illegible or & & \\
$\quad$ incomplete & 230 & $(7)$ \\
Duration & 221 & $(7)$ \\
Formulary or blacklist & 219 & $(7)$ \\
Therapeutic drug level monitoring or & & \\
pharmokinetics & 216 & $(7)$ \\
Adverse drug reactions & 155 & $(5)$ \\
Interaction or incompatibility & 52 & $(2)$ \\
Pharmacology & 171 & $(5)$ \\
Miscellaneous & 13 & $(<1)$ \\
Not stated & 3758 & $(115)$ \\
\hline Total & & \\
^As percentage of 3273 incidents. & &
\end{tabular}

counted as monitoring incidents. A specifically designed form was used, which had been previously piloted in four hospitals. The form was a "tick the box" format with additional space for free text (fig 1), and information to help staff complete the form was included on its reverse side. A pharmacist in each of the study hospitals was appointed as a survey coordinator and was briefed about data collection and, in turn, briefed his or her colleagues.

Pharmacy staff recorded details of where the PMIs were identified and by whom, the nature of the incident, and the outcome of the PMI. The time spent seeking additional information, resolving the problem, and communicating with the relevant staff was recorded. If the problem was referred to a drug information centre, the time its staff spent seeking the information was not included. Ward, dispensary, residency services (where present), and on call activity was included. Additional information about the wards and hospitals was recorded by the ward pharmacists, including the number of beds in each ward. This was used as a measure of workload according to the occupied plus available beds in the ward at the time of the survey. RB examined the completed forms for accuracy, completeness, and clarity; any ambiguities were clarified before the data were entered on to Excel spreadsheets for analysis.

\section{Results}

Two hundred and ten pharmacists were involved in monitoring prescriptions in 489 wards with 10337 beds. A total of 3273 PMIs were recorded from the 31 hospitals (mean 106/hospital (range 3-301, median 89)); 23

Table 2 Reported outcome for 3273 prescription monitoring incidents identified

\begin{tabular}{lrr}
\hline Outcome & No & (\%) \\
\hline Prescription altered & 1611 & 49 \\
Information only & 580 & 18 \\
Incident resolved without intervention & 443 & 14 \\
Prescription unchanged, advice & 403 & 12 \\
$\quad$ accepted & 81 & 3 \\
Prescription unchanged, advice not & & \\
$\quad$ accepted & 132 & 4 \\
Unt specified & 23 & $<1$ \\
\hline
\end{tabular}

Table 3 Number of prescription monitoring incidents $/ 100$ beds/week by specialty

\begin{tabular}{lcc}
\hline Specialty & $\begin{array}{c}\text { Median (range) } \\
\text { PMIs/100 beds/week }\end{array}$ & $\begin{array}{c}\text { No of } \\
\text { hospitals }\end{array}$ \\
\hline Intensive therapy units $_{\text {Special }^{\dagger}}$ & $75(0-375)$ & 24 \\
Medicine $_{\text {Geriatrics }}$ & $33(0-83)$ & 11 \\
Surgery & $32(5-80)$ & 25 \\
Paediatrics & $29(6-88)$ & 20 \\
Psychiatry & $21(3-73)$ & 27 \\
Obstetrics & $12(0-89)$ & 24 \\
\end{tabular}

^Those reporting dedicated wards for that specialty and receiving a pharmacy visit.

Including various specialty wards such as haematology, oncology, neurology, etc.

were unresolved at the end of the study. In all, $2706(83 \%)$ were identified by a pharmacist, the remainder were identified by other pharmacy staff $(137,4 \%)$ or initiated by the clinician $(174,5 \%)$, nurse $(185,6 \%)$, or others $(71,2 \%)$. Of the total PMIs, $2718(83 \%)$ occurred in wards and $555(17 \%)$ in dispensaries, on call, or in sterile production units. The total time entailed in resolving 2935 PMIs in which time had been noted was 242 hours and 24 minutes. Pharmacists spent a mean of 5.0 minutes for each PMI, from identifying it to resolving it; times over five minutes tended to be rounded to the nearest five minutes.

Table 1 shows the nature of the PMIs. Pharmacists could choose more than one category, so the total of 3758 was greater than the total number of PMIs. Table 2 shows the reported outcomes for the 3273 problems identified. Pharmacists provided advice to clinicians on 2095 occasions; in 2014(96\%) the advice was accepted and in $1611(77 \%)$ resulted in a change in the prescription. Pharmacists investigated 443 PMIs and were satisfied that no more action should be taken after further checking. A performance indicator of PMIs/100 beds/week was derived. Table 3 shows this performance indicator by specialty; the median values ranged from three for obstetric wards to 75 for intensive therapy units. The range of values for intensive therapy units at each site was $0-375$, which resulted partly from extrapolation from a small number of beds at each site. The median value of PMIs/100 beds/week for a hospital was 29.8 (range 3.6-82.1). Figure 2 shows the results for each hospital with the teaching and special hospitals identified.

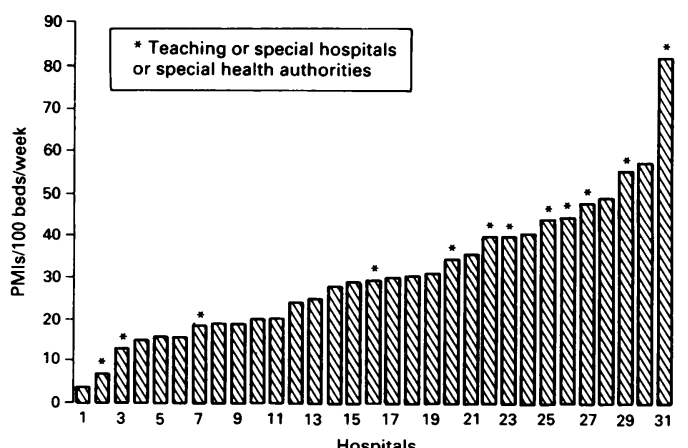

Fig 2 Prescription monitoring incidents (PMIs)/100 beds/week for each hospital in North West Thames region, Fune 1990 


\section{Discussion}

This study is the largest survey of prescription monitoring by pharmacists in the United Kingdom. It shows the extent to which pharmacists influence prescriting at ward level and that this is related to the fundamentals of prescribing such as the choice of dose, frequency, drug, and route of administration. A performance indicator of prescription monitoring incidents $/ 100$ beds/week was calculated and showed a 20-fold range between sites, suggesting different levels of activity by pharmacists.

The high level of acceptance of the pharmacists' advice by prescribers suggests that the advice was valid and that the pharmacists were making a real contribution to patient care. Most PMIs were related to the quality of basic prescription writing, such as the correct dose, drug, or route of administration. This finding is similar to those of previous studies. ${ }^{7-9} 11$ Surprisingly, about one in seven PMIs was related to the choice of treatment, usually considered to be the domain of doctors. Most of these incidents related to antibiotic prescribing, pain control, diuresis (usually in response to serum potassium concentrations), total parenteral nutrition, and skin or wound care. There was a low incidence of PMIs relating to adverse drug reactions $(7 \%)$ and to drug interactions $(5 \%)$; whether this indicates a low incidence of these events or a training need in the pharmacists is unclear, although Cairns and Prior showed that $15 \%$ of their interventions with prescribers were related to adverse drug reactions. ${ }^{12}$ Incidents related to therapeutic drug level monitoring and pharmacokinetics nearly all came from hospitals in which the pharmacy offered a therapeutic drug level monitoring service. The interventions from pharmacists which are sometimes thought to be bureaucratic, such as challenging an incorrectly written prescription for a controlled drug or the prescribing of a nonformulary drug, represented a small proportion of the total number of interventions.

Of the 2095 cases when a pharmacist advised a doctor about a prescription or asked for it to be changed, the advice was rejected in $4 \%$ of the incidents and the prescription changed in $77 \%$. When the PMI was related to the choice of treatment the results were similar ( $6 \%$ were rejected, $72 \%$ led to the prescription being changed). In 1985 Ross reported that $6 \%$ of pharmacists' suggestions were rejected; ${ }^{7}$ Hawkey et al reported acceptance of $83 \%,{ }^{9}$ and Klopfer and Einarson, reviewing 23 studies in the United States, showed a mean rate of acceptance of $85 \% .^{6}$ There may have been a tendency for pharmacists not to attempt interventions when they thought that the chance of their advice being accepted was small; the relatively high degree of unanimity in the studies, however, and our own experience suggests that this is not a major influence.

The results of this survey suggest that pharmacists are conducting a regular process of improving prescribing through talking with the prescriber, usually a junior doctor. This information on prescribing practice, however, is not fed back into the prospective influences on prescribing such as medical audit, the drug and therapeutics committee, formularies, and prescribing guidelines. There needs to be a route for this to happen. Currently, we are studying models of audit in which the pharmacists' interventions have been used to identify suitable areas for a medical audit of prescribing. The pharmacists and clinicians then agree on standards, and the pharmacists monitor practice against those standards as part of their daily ward pharmacy service. This model is applicable to all types of beds and, as the prescribing and administration of drugs should not be separated, could be extended to clinical audit involving nurses and other professions.

The second objective of this study is assessing the uniformity of performance of the pharmacists across the region. The performance indicator of $\mathrm{PMIs} / 100$ beds/week shows a wide range, but can it be related to the quality of the prescription monitoring service? Other major sources of variation could be the quality of the prescribers, patient mix, and patient turnover. There is some evidence that this performance indicator does give an indication of quality. Hospitals above the median have more pharmacists with postgraduate qualifications in clinical pharmacy than those below it; teaching hospitals are above the median. The lowest recorded performance indicator was from a hospital pharmacy staffed by one locum pharmacist, who left soon after the survey but before the results were known. When the results were analysed by specialty the greatest number of PMIs/100 beds/week occurred in specialties with the greatest use of drugs and variety of regimens. Finally, the order of the hospitals in terms of performance broadly fitted with the regional specialists' expectations.

The use of the number of beds as the denominator combined convenience and accuracy as the pharmacists could quickly count them; bed occupany, however, could vary, and occupied beds were used when this work was repeated in 1991. An alternative would be the number of patients or prescription sheets seen. The number of patients allows for variations in patient turnover but is harder for the pharmacist to record; ascertaining the number of prescription sheets is complicated by the absence of drug charts from wards (for example, when they have been sent with the patient to operating theatres). Further work is needed to relate the above variables. In addition, a weighting system based on the number of specialty beds is currently being investigated.

The district pharmaceutical officers accepted PMIs/100 beds/week as a valid regional performance indicator. The study was repeated in 1991 and is being repeated annually, the results reaching the hospitals within three months. In addition, the regional specialists in clinical pharmacy are visiting all 
districts annually to help monitor, plan, and develop clinical pharmacy services. Information from the regional PMI survey provides useful information to support this function. An integrated system is possible in which managers monitor their PMIs routinely and a sample of their aggregated data could be sent to region annually.

This survey made no attempt to measure the quality of the PMIs, although it was possible to form an opinion from the text part of the recording form. Several authors have attempted to measure the quality of pharmacists' interventions; Cousins and Hatoum recently reported a software based system in which pharmacists enter their interventions into a computer on their return from the wards, for subsequent grading by a senior pharmacist. ${ }^{10}$ This, or an adaptation of our own system, produces a method by which local managers can monitor the performance of their ward pharmacists and identify training needs.

This study has shown the large role that pharmacists have in affecting prescribing at ward level. The information is collected as part of the daily ward pharmacy activities and may form the basis of an integral medical audit system in which pharmacists and clinicians collaborate in setting standards and monitoring prescriptions. The proposed performance indicator of $\mathrm{PMI} / 100$ beds/week seems to form a pragmatic system which may have a value at regional level for comparing provider units and trusts; further work is needed, however, to refine the performance indicator and show a relation with quality of service. The wide range of values of the indicator among pharmacy departments suggests that pharmacists need to develop audit of their own profession.

We thank all the local organisers who took part in the study, Andy Blackett for software development, and Noreen Butle for data entry and typing this manuscript.

1 Department of Health. Health services management. The way forward for hospital pharmaceutical services. London: $\mathrm{DOH}$ 1988. (HC(88)54.)

2 Barber ND. Is "safe, effective and economic" enough? Pharmaceutical fournal 1991;246:671-2.

3 Willson A. A new approach to prescription monitoring by ward pharmacists. Pharmaceutical fournal 1983;230: 78-80

4 Fahey M, Oakley PA, Sims K. Prescription monitoring and the oncology patient. Pharmaceutical fournal the oncology

5 Batty R, Barber ND. Prescription monitoring for ward pharmacists. Pharmaceutical fournal 1991;247:242-4.

6 Klopfer JD, Einarson TR. Acceptance of pharmacists' suggestions by prescribers: a literature review. Hospital Pharmacy 1990;25:830-2, 834-6.

7 Ross AJ. Evaluating the benefits of a clinical pharmacy service. Pharmaceutical fournal 1985;234:683-5.

8 Ross AJ. Quantifying ward pharmacy and its impact on patients. British fournal of Pharmaceutical Practice 1987;9:267-73.

9 Hawkey CJ, Hodgson S, Norman A, Daneshmend TK, Garner ST. Effect of reactive pharmacy intervention on the quality of hospital prescribing. $B M F$ 1990; 300:986-90.

10 Cousins D, Hatoum H. The development of computerised quality assurance system for clinical computerised quality assurance system

11 Marchand HC. Implementing a quality assurance program. Am f Hosp Pharm 1987;44:60-2.

12 Cairns CJ, Prior EGR. The clinical pharmacist: a study of his hospital environment. Pharmaceutical fournal 1983, 230: 16-8. 